\title{
Black phosphorus quantum dots: A new-type of water-based high-efficiency lubricant additive
}

\author{
Weiwei TANG ${ }^{1}$, Zhiqiang JIANG ${ }^{1}$, Baogang WANG ${ }^{2}$, Yufeng LI $^{1, *}$ \\ ${ }^{I}$ School of Biological and Chemical Engineering, Panzhihua University, Panzhihua 617000, China \\ ${ }^{2}$ College of Chemistry and Chemical Engineering, Southwest Petroleum University, Chengdu 610500, China \\ Received: 13 May 2020 / Revised: 11 June 2020 / Accepted: 18 July 2020 \\ (C) The author(s) 2020 .
}

\begin{abstract}
Black phosphorus quantum dots (BPQDs), obtained via a typical solution-based top-down method, were used as water-based lubricant additives. BPQDs exhibited remarkable friction reduction and anti-wear properties even at the ultra-low concentration of $0.005 \mathrm{wt} \%$, which reduced the friction coefficient and wear volume of the base liquid by $32.3 \%$ and $56.4 \%$, respectively. In addition, the load-supporting capacity of the base liquid increased from $120 \mathrm{~N}$ to over $300 \mathrm{~N}$. BPQDs-based additives exhibited a relatively long lifetime at a relatively high load of $80 \mathrm{~N}$. The performance of BPQDs considerably exceeded that of the BP; this may be attributed to their small and uniform particle size, good dispersion stability in water, and high reactivity at the frictional surfaces. The results of the surface wear resistance analysis demonstrated that a robust tribochemical film with a thickness of approximately $90 \mathrm{~nm}$ was formed on the rubbing surface lubricated with $0.005 \mathrm{wt} \%$ of BPQDs dispersion. Moreover, the film served as a direct evidence of the excellent tribological performance of BPQDs.
\end{abstract}

Keywords: black phosphorus quantum dots; water-based lubricant additives; tribological performance; lubrication mechanism

\section{Introduction}

In recent years, sustainable development has been a commonly discussed theme worldwide, which is increasingly driving people's attention toward protecting the environment and saving energy. Recently, water-based lubricants have been associated with sustainable development because of their many advantages such as low cost, good recyclability, high cooling capability, and environmental friendliness [1]. However, water itself is a poor lubricant because of its low viscosity, poor film-forming ability, and high corrosivity. Several studies have shown that the most efficient approach for improving the lubrication ability of water-based lubricants, with respect to friction reduction and anti-wear performance, entails additives.

Two-dimensional (2D) layered nanomaterials, such as transition metal dichalcogenides (TMDs) $[2,3]$, carbon nitride $\left(\mathrm{C}_{3} \mathrm{~N}_{4}\right)$ [4], graphene [5, 6], zirconium phosphate [7], palygorskite nanoplatelets [8], hexagonal boron nitride (hBN) [9], and MXene $\left(\mathrm{Ti}_{3} \mathrm{C}_{2}\right)$ [10], have demonstrated high potential in the field of water-based lubricant additives [11-13]. Using these nanomaterials as additives can effectively enhance friction reduction and anti-wear performance in lubricants; however, they also have several shortcomings, including poor dispersion stability, non-uniform particle sizes, and poor embedding stability between the rubbing surfaces [14, 15]. These shortcomings lead to low tribological properties in traditional 2D nanomaterials, thus restricting

* Corresponding author: Yufeng LI, E-mail: lyfpzh@163.com 
their prospective applications in water-based lubrication. Therefore, exploring novel 2D nanoadditives with a uniform particle size distribution, good dispersion stability in water, and high stability on the rough surfaces is needed. As a new type of $2 \mathrm{D}$ layered nanomaterial, black phosphorus (BP) has attracted considerable attention because it has promising applications in tribology owing to its excellent physicochemical and mechanical properties $[16,17]$. Seminal works have demonstrated that BP and its composites are useful as lubricant additives and can provide excellent lubricating effects owing to their superior properties [18-23]. However, BPbased materials are not frequently used as waterbased lubricant additives owing to their low dispersion stability in water. Ultra-miniaturization, and nanocrystallization in particular, are effective methods for improving this property in BP-based materials.

Black phosphorus quantum dots (BPQDs) first prepared by the top-down method are novel derivatives of BP [24]. BPQDs have attained growing expectations in material and chemical sciences because they have applications in various fields such as bioimaging [25, 26], biosensing [27-29], drug delivery [30], photodynamic therapy [31, 32], solar cells [33], photocatalysis [34], and electrocatalysis [35]; and as photovoltaic devices [36]. The use of zero-dimensional (0D) carbon quantum dots (CQDs) and transition metal dichalcogenides quantum dots (TMDQDs) as additives can efficiently improve the lubrication performance of water-based lubricants [37-41]. Moreover, BPQDs also have high potential as high-performance water-based lubricant additives, since they have a uniform size distribution, good dispersion stability in water, desired reactivity, and excellent mechanical properties. Luo et al. studied BPQDs in aqueous ethylene glycol (EG). The BPQDs/ EG suspensions exhibited a unique superlubricity phenomenon induced by the excellent tribological properties of BPQDs [42]. However, studies on using BPQDs as water-based lubricant additives are limited; hence, novel research on BPQDs nanoadditives is required.

In this study, BPQDs were obtained by the solutionbased top-down method, using bulk BP powder as the precursor. The morphology, structure, and composition of the as-synthesized BPQDs were comprehensively analyzed using various microscopic and spectroscopic techniques. The BPQDs exhibited good dispersion stability in water; hence, they were used as water-based lubricant additives. The tribological characteristics of the BPQDs were evaluated using a ball-on-plate standard tribometer operating in a linear reciprocating mode with steelsteel contact. The effects of additive concentration, load, and test duration on the tribological properties of BPQDs were investigated. A reasonable lubrication mechanism of BPQDs was confirmed by scanning electron microscopy (SEM), energy-dispersive spectroscopy (EDS), X-ray photoelectron spectroscopy (XPS), and cross-sectional Transmission electron microscopy (TEM). This study presents potential novel and significant applications of BPQD-based lubricant additives.

\section{Experimental section}

\subsection{Chemicals}

BP powder was obtained from Nanjing XFNANO Material Technology Co., Ltd. (China), and further stored in a dark Ar-glovebox. N-methyl-2-pyrrolidone (NMP, 98.5\%) and triethanolamine (TEA, 99.5\%) were obtained from Sigma-Aldrich Chemical Reagent Co., Ltd. (China) and Beijing Macklin Chemical Reagent Co., Ltd. (China), respectively. Petroleum ether (60\%-90\%) was obtained from Chengdu Kelong Chemical Co., Ltd. (China). All chemicals and reagents were used without further purification. Ultrapure water with a resistivity of approximately $18.25 \mathrm{M} \Omega$ $\mathrm{cm}$ was obtained by an ultrapure water purifier (China).

\subsection{Preparation of BPQDs}

As shown in Fig. 1, BPQDs were synthesized by the solution-based top-down method, that is, the ultrasonic method [24]. Typically, $30 \mathrm{mg}$ of BP power and $3.0 \mathrm{~mL}$ of NMP were grounded for 20 min in an agate mortar. The mixture was rapidly transferred to a $50 \mathrm{~mL}$ sealed conical flask, containing $30 \mathrm{~mL}$ of NMP, and was sonicated for $3 \mathrm{~h}$ 


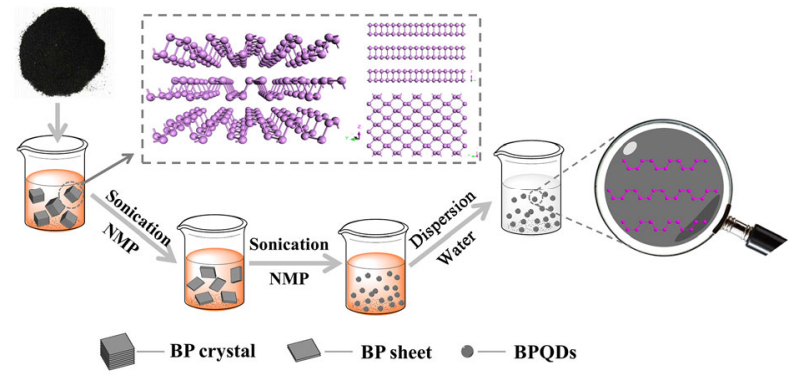

Fig. 1 Schematic of BPQDs synthesis.

(ultrasonic power: 1,200 W). The resultant black suspension was further sonicated in an ultrasonic bath that was kept below $0{ }^{\circ} \mathrm{C}$ in an ice bath for $8 \mathrm{~h}$ (ultrasonic power: $360 \mathrm{~W}$ ). Then, the resulting black suspension was centrifuged at 7,000 rpm for $20 \mathrm{~min}$, and the supernatant containing BPQDs was slowly decanted into the dialysis bag (molecular weight cutoff: 1,000 Da). The residual NMP molecules in the supernatant were removed using the dialysis method as efficiently as possible. The dialyzate was centrifuged at 16,000 rpm for $20 \mathrm{~min}$, and the BPQDs were collected in the precipitate.

\subsection{Preparation of BPQDs and BP dispersions}

Triethanolamine (TEA) has been widely used as a corrosion inhibitor in water lubrication. Hence, the base liquid used to prepare the water-based lubricants was a TEA aqueous solution (2.0 $\mathrm{wt} \%$ ). The synthesized BPQDs were quantitatively dispersed into the base liquid by ultrasonic treatment for $10 \mathrm{~min}$ to obtain a $0.1 \mathrm{wt} \%$ BPQDs dispersion. Various concentrations of BPQDs dispersions (0.001-0.02 wt $\%$ ) were obtained by diluting the $0.1 \mathrm{wt} \%$ of BPQDs dispersion with the base liquid. As the reference samples, BP dispersions were prepared by the described method.

\subsection{Characterization}

Transmission electron microscopy (TEM) and atomic force microscopy (AFM) were performed to observe the morphology and microstructure of the synthesized BPQDs. TEM and HR-TEM images of BPQDs were collected using a Tecnai G2 TF20 system at an accelerating voltage of $200 \mathrm{kV}$. The specimen was prepared by drop-casting a BPQDs aqueous suspension $(0.05 \mathrm{mg} / \mathrm{mL})$ onto a carbon-coated copper grid, followed by drying at room temperature. AFM images were obtained using a Multimode 8 system (Bruker), and the specimen was prepared by dropcasting the sample aqueous solution onto a $\mathrm{Si}$ substrate, followed by drying at room temperature. The tests were performed under tapping mode in air Fourier transform infrared (FTIR) spectra were recorded at wavenumbers in the range of 400 $4,000 \mathrm{~cm}^{-1}$ on a WQF-520 spectrophotometer using the $\mathrm{KBr}$ pellet technique. X-ray diffraction (XRD) measurements were performed using a D8 Advance X-ray diffractometer with a wavelength $(\lambda)$ of $0.15418 \mathrm{~nm}$. BP and BPQDs samples were characterized by Raman spectroscopy (JY-HR800 microscope with $532 \mathrm{~nm}$ laser excitation). Additionally, the particle diameter and height of BPQDs were measured using a Nicomp 380 Dynamic Light Scattering Particle Size Analyzer and AFM, respectively. Images of the BPQDs dispersions were obtained using a Canon camera (EOS 550) under sunlight, and the fluorescence spectra were recorded using an LS-55 spectrofluorometer.

\subsection{Tribological tests}

The tribological behaviors of the BPQDs dispersions were systematically evaluated using a UMT tribometer (Bruker, USA) in linear reciprocating mode. In particular, a commercially available AISI52100 steel ball $(12.7 \mathrm{~mm}$ in diameter and hardness of approximately 59-61 HRC) reciprocally slid against a low stationary AISI-52100 steel plate $(50 \mathrm{~mm} \times$ $40 \mathrm{~mm} \times 3 \mathrm{~mm}$ in size and approximately 59-61 HRC in hardness). The steel plate was mounted in a holder and connected through a stiff lever coupled with a friction force transducer. The friction coefficients (FC) were automatically recorded using a computer coupled with a high-sensitivity sensor. The tribological tests were performed by drop-casting approximately $0.5 \mathrm{~mL}$ water-based lubricant onto the contact area of the lower steel plate. Each test was conducted three times under specific test conditions (loads of 40-300 N, test duration within the range of 20$120 \mathrm{~min}$, linear velocity of $10 \mathrm{~mm} / \mathrm{s}$, amplitude of $5 \mathrm{~mm}$, and temperature of $30^{\circ} \mathrm{C}$ ) to obtain a friction coefficient (FC) curve. The mean friction coefficient (MFC) value was calculated as the average of all the data points on the FC curve, considering the 
error bars.

\subsection{Wear track surface analysis}

After the friction tests, the wear track surfaces on the lower plates were adequately washed by petroleum ether $(60 \%-90 \%)$ under ultrasonic treatment for 10 min. The width, depth, mean wear volume (MWV), two-dimensional (2D) profile, and three-dimensional (3D) morphology of the wear tracks of the lower plates were collected using a Bruker Contour GT-K 3D optical microscope. The morphologies and element compositions of the wear track surfaces were obtained via SEM coupled with EDS and XPS, respectively. To detect the tribofilm on the wear track surface, an ultra-small and thin cross-section was pulled out from the wear track of the lower plate via the focused ion beam (FIB) technique, and it was characterized by TEM coupled with EDS.

\section{Results and discussion}

\subsection{Characterization of BPQDs}

As shown in Fig. 2(a), TEM confirmed that BPQDs with a uniform size distribution were successfully synthesized by the solution-based top-down method. The particle size distribution shown in Fig. 2(c) indicates that the average particle size of BPQDs was approximately $3.9 \mathrm{~nm}$. High-resolution transmission electron microscopy (HR-TEM) in Fig. 2(b) demonstrates that the prepared BPQDs has good crystallinity, and the spacing of the lattice fringes of the BPQDs was approximately $0.21 \mathrm{~nm}$, which can be assigned to the (020) plane of the BP crystal. As illustrated in Figs. 2(d-f), the average height of the BPQDs was $1.0 \pm 0.4 \mathrm{~nm}$, as obtained by AFM and 3D AFM. It can be inferred that the BPQDs were not spherical nanoparticles, and the rounded nanosheets were similar to graphene quantum dots.
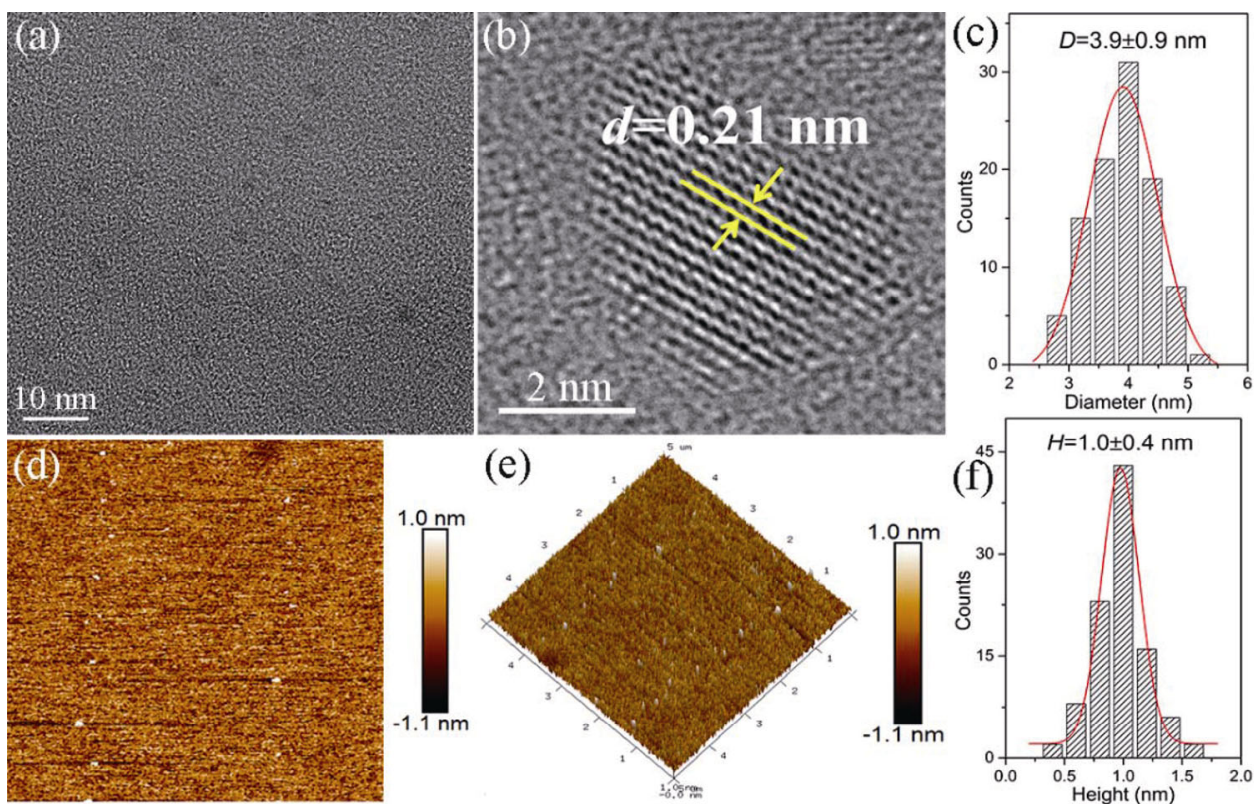

(e)
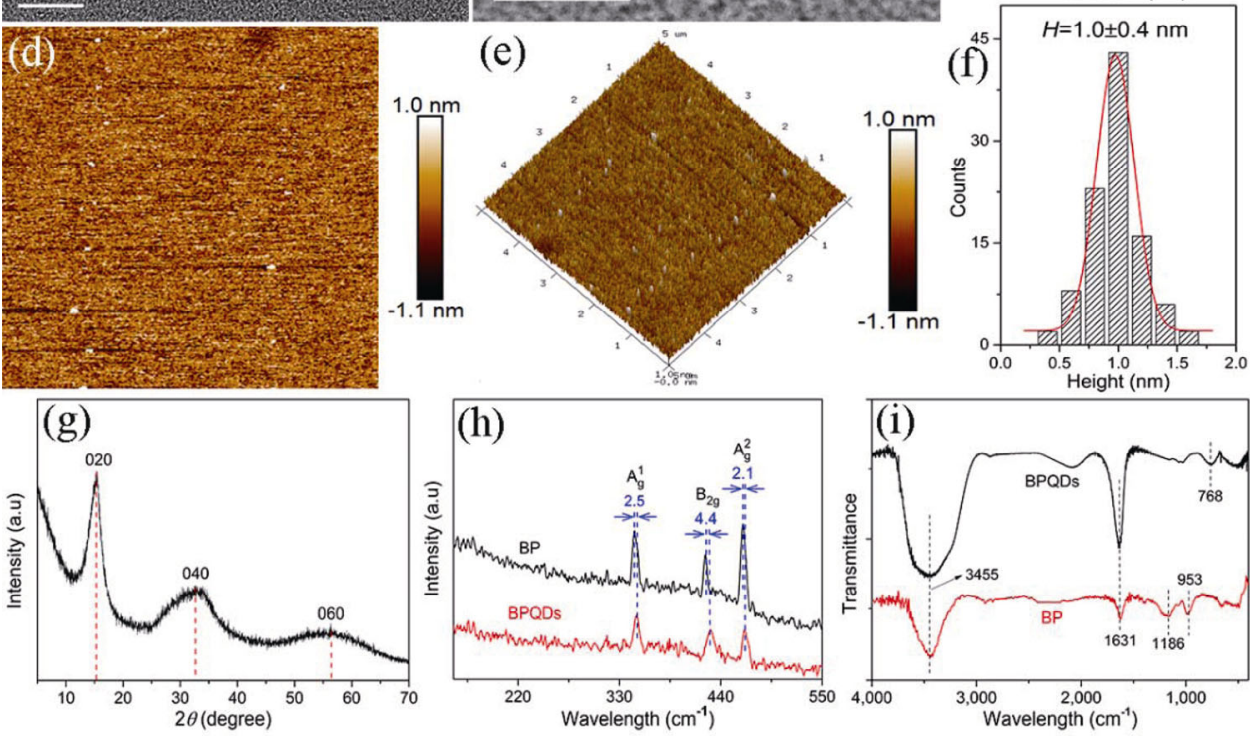

Fig. 2 Characterization of BPQDs: (a) TEM and (b) HR-TEM images; (c) diameter and (f) height distributions; (d) AFM and (e) 3D AFM images; (g) XRD patterns; (h) Raman and (i) FTIR spectra of BP and BPQDs. 
As shown in Fig. 2(g), the XRD pattern of BPQDs exhibited three typical peaks centered at $15.4^{\circ}$, $32.6^{\circ}$ and $56.5^{\circ}$ that are related to (020), (040) and (060) planes of the BP crystal [28, 34]. The sharp diffraction peak at $15.4^{\circ}$ was consistent with the HR-TEM image (Fig. 2(b)), and confirmed the good crystallinity of the BPQDs. In Fig. 2(h), the Raman spectrum of BP had three characteristic peaks located at 354, 433, and $466 \mathrm{~cm}^{-1}$, which were attributed to the out-of-plane phonon mode $\left(A_{g}^{1}\right)$ and the in-plane modes $\left(B_{2 g}\right.$ and $\left.A_{g}^{2}\right)$ of the BP crystal [32, 43], respectively. Compared to the $\mathrm{BP}$ sample, the above peaks for BPQDs exhibited obvious blue shifts of 2.5, 4.4, and $2.1 \mathrm{~cm}^{-1}$ because of their small particle size and abundant surface functional groups. In addition, FTIR spectra were used to analyze the surface characteristic groups of the BP and BPQDs samples. In particular, the four feature peaks at 3,455, 1,631, 1,186, and $953 \mathrm{~cm}^{-1}$, shown in Fig. 2(i), can be attributed to the $\mathrm{O}-\mathrm{H}, \mathrm{C}=\mathrm{O}, \mathrm{P}=\mathrm{O}$, and $\mathrm{P}-\mathrm{O}$ stretching vibrations of hydroxyl, carbonyl and phosphorus oxide, respectively. The above peaks were derived from the absorption of water and $\mathrm{CO}_{2}$ as well as the surface oxidation of BP sample. The intensities of the peaks of $\mathrm{O}-\mathrm{H}\left(3,455 \mathrm{~cm}^{-1}\right)$ and $\mathrm{C}=\mathrm{O}\left(1,631 \mathrm{~cm}^{-1}\right)$ in the spectrum of BPQDs is considerably stronger than that of BP sample. Therefore, these strong peaks are caused by oxygencontaining groups on the surfaces of BPQDs and $\mathrm{CO}_{2}$ adsorption. In addition to the aforementioned peaks, a new peak at $783 \mathrm{~cm}^{-1}$ induced by the asymmetric stretching vibration of $\mathrm{C}-\mathrm{N}$ can also be observed, demonstrating that the synthesized BPQDs were modified by some NMP molecules. The abundant oxygen-containing groups and NMP molecules are expected to endow BPQDs with good dispersion stability in water.

\subsection{Evaluation of the dispersion stability of BPQDs in the base liquid}

The dispersion stability of nanomaterials as lubricant additives is a crucial factor to consider. It was systematically evaluated for the BPQDs aqueous dispersion $(0.005 \mathrm{wt} \%)$ by macroscopic observation and fluorescence spectrophotometry. Figure 3 (left side) shows that a light brown dispersion was obtained for the BPQDs dispersion. No agglomerates or precipitates were observed at the bottom of the container, even when the dispersion was stored for two weeks. These results reflect the good dispersion stability of the BPQDs in water. It is well known that a good dispersion of BPQDs could emit bright fluorescence under UV radiation because of the quantum confinement and surface passivation effects, whereas the fluorescence will be attenuated or even quenched due to the aggregation of BPQDs [39, 44, 45]. The photoluminescence (PL) excitation and emission spectra in Fig. 3(a) demonstrated that the BPQDs dispersion emitted blue fluorescence at $440 \mathrm{~nm}$, under $370 \mathrm{~nm}$ excitation. The bright blue fluorescence could be observed from the image of BPQDs dispersion (insets) under UV irradiation (365 nm). Although the PL emission spectrum exhibited a slight blue shift, the fluorescence intensity did not have any variation when the dispersion was analyzed after the sample settled for two weeks (Fig. 3(b)), indicating that BPQDs had an excellent dispersion stability in the base liquid at a micro-level.

\subsection{Tribological properties of BPQDs as water- based additives}

The effects of the additive concentration $(c, w t \%)$,
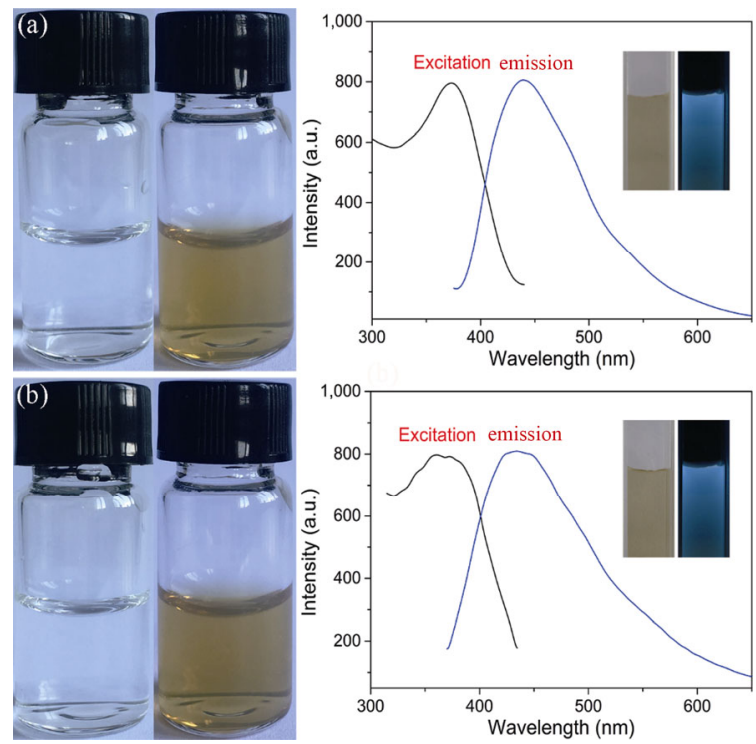

Fig. 3 Images of the base liquid and the BPQDs dispersion (0.005 wt $\%$ ), as along with the PL excitation and emission spectra of the $0.005 \mathrm{wt} \%$ BPQDs dispersion (a) after preparation and (b) after a period of two weeks. 
on the lubrication performance of BPQDs, were investigated in detail. Figure 4(a) shows that the FC curves of BPQDs dispersions at different additive contents $(c)$ were smoother and were located below, when compared to that of the base liquid, indicating the outstanding friction-reducing effect of BPQDs. The base liquid containing $0.005 \mathrm{wt} \%$ of BPQDs exhibited the lowest FC curve, suggesting that there may be an optimal $c$ for BPQDs in the base liquid. Furthermore, the optimal $c$ for BPQDs in this study was $0.005 \mathrm{wt} \%$, which is the lowest additive content $(c)$ reported in the literature for nano-additives (including metal- [46], metal oxide-[47-49], metal sulfide-[50, 51], metal borate-[52], polymer-[53], and carbon-based nano-additives [54-56]). As shown in Fig. 4(b), the MFC and MWV of the lower plates first decreased drastically and then had a slight increase when increasing $c$, further confirming an optimal $c$ of $0.005 \mathrm{wt} \%$ for this study. The addition of BPQDs $0.005 \mathrm{wt} \%$ to the tested sample reduced the MFC and MWV of the base liquid by 32.3\% and $56.4 \%$, respectively.
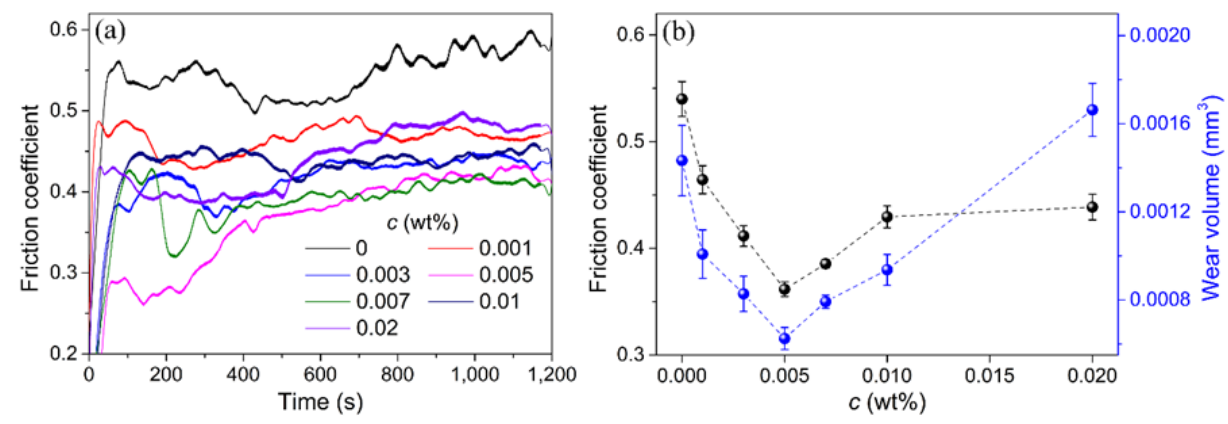

Fig. 4 (a) FC curves (smoothed) of BPQDs dispersions with different additive content, $c$ in BPQDs wt\%. (b) MFC and MWV of lower plates lubricated by BPQDs dispersions as a function of $c$. (Test conditions: $5 \mathrm{~Hz}, 20 \mathrm{~min}, 40 \mathrm{~N}$, and room temperature).
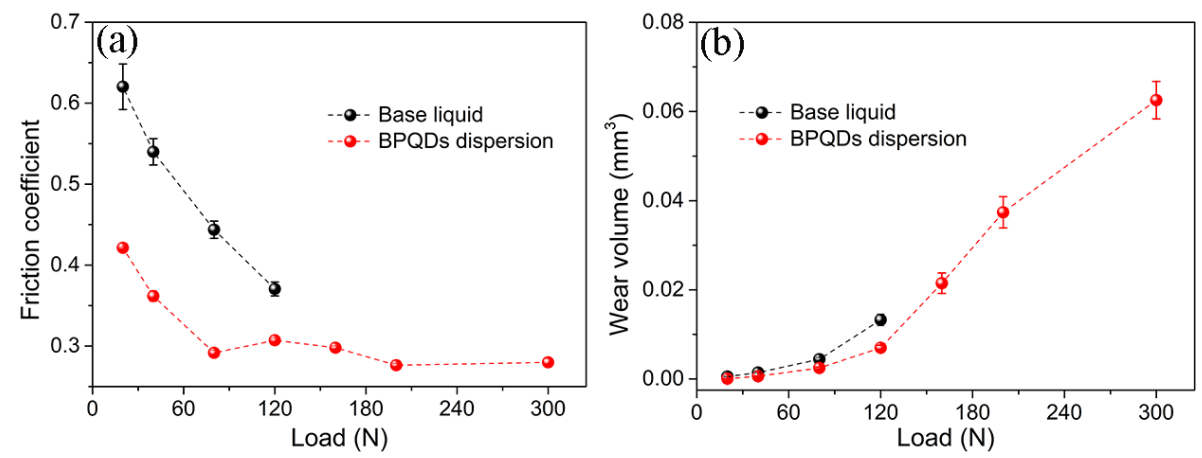

Fig. 5 (a) MFC and (b) MWV of lower plates lubricated by the base liquid and the BPQDs dispersion (0.005 wt\%), as a function of load. (Test conditions: $5 \mathrm{~Hz}, 20 \mathrm{~min}$, and room temperature). 
For comparison purposes, BP powder was also utilized as additive of the base liquid to further highlight the superior performance of BPQDs as additives. In Fig. 6(a), the values of the FC curve for the BPQDs dispersion (0.005 $\mathrm{wt} \%)$ are remarkably smaller than those of the FC curve for BP dispersion $(0.005 \mathrm{wt} \%)$ and for the base liquid. Likewise, the variation tendency of the MFC and MWV (Fig. 6(b)) was highly consistent with the FC curves, illustrating that the friction-reducing and anti-wear effects of the ultra-small BPQDs were significantly better than those of the large-sized BP. Nonetheless, BP as an additive can moderately promote the lubrication performance of the base liquid. The excellent tribological properties of BP-based additives might be closely related to their sizes and geometrical shapes. It is well accepted that the lifetime of lubricant additives has great significance on their practical application. Herein, the lifetime of BPQDs was assessed by prolonging the duration of the friction tests from 20 to $120 \mathrm{~min}$ at a relatively high load $(80 \mathrm{~N})$. As shown in Fig. 6(c), although the FC curves of the BPQDs dispersion $(0.005 \mathrm{wt} \%)$ and the base liquid first showed a slight increase and then a high decrease throughout the friction process, the former was distinctly lower than the latter at the second half of the friction test. In addition, Fig. 6(d) shows that the $0.005 \mathrm{wt} \%$ BPQDs dispersion resulted in reductions of MFC and MWV by $27.6 \%$ and $41.2 \%$, respectively, compared with the base liquid. Thus, BPQDs could maintain their lubrication effect without decreasing over a long period at relatively high loads; i.e., the BPQDs as water-based lubricant additives have a long lifetime.

\subsection{Wear track surface analyses and lubrication mechanism}

Research on the applications of BPQDs in the field of nano-additive lubricants is still growing. As a new nano-additive, it is particularly important to explore the lubrication mechanism of BPQDs. In this study, the wear tracks of the lower plates were analyzed by 3D optical microscope, SEM coupled with EDS, XPS, and cross-sectional TEM characterization. Figure 7 shows the 3D morphologies and
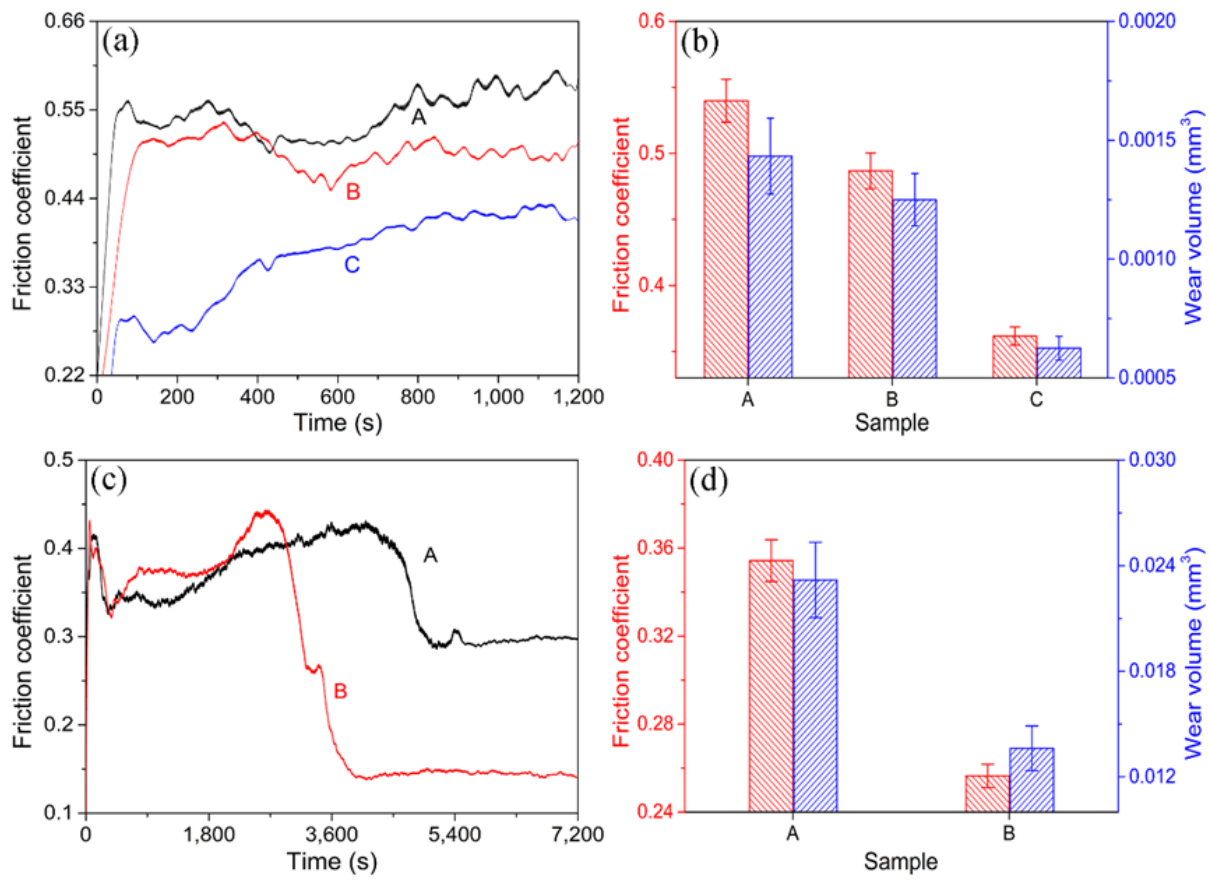

Fig. 6 (a) FC curves (smoothed) and (b) MFC and MWV of lower plates lubricated by: (A) the base liquid, (B) 0.005 wt $\%$ of $\mathrm{BP}$, and (C) $0.005 \mathrm{wt} \%$ of BPQDs dispersions (test conditions: $5 \mathrm{~Hz}, 20 \mathrm{~min}, 40 \mathrm{~N}$, and room temperature). (c) FC curves (smoothed) and (d) MFC and MWV of lower plates lubricated by: (A) base liquid and (B) 0.005 wt $\%$ of BPQDs dispersion (test conditions: $5 \mathrm{~Hz}, 120 \mathrm{~min}, 80 \mathrm{~N}$, and room temperature). 
2D profiles of the wear tracks of lower plates lubricated by the base liquid, BP, and BPQDs dispersions (0.005 wt \%). The wear track lubricated by the base liquid had a width of $0.2572 \mathrm{~mm}$ and a depth of $0.0029 \mathrm{~mm}$, which are very large values, as well as many deep scratches (Fig. 7(a)), implying that serious adhesive and/or corrosion wear occurred during the friction process owing to the poor lubrication capacity of the base liquid. This situation was only slightly improved when the $c$ of BP was $0.005 \mathrm{wt} \%$ in the base liquid (Fig. 7(b)). In contrast, the width and depth of the wear track lubricated by the BPQDs dispersion (0.005 wt\%) remarkably reduced to 0.2326 and $0.0014 \mathrm{~mm}$, respectively (Fig. 7(c)). Furthermore, the wear track depth of the base liquid decreased by $51.7 \%$ when adding the low $c$ of BPQDs $(0.005 \mathrm{wt} \%)$. These results were consistent with the microscopic images of the wear tracks (Fig. S1 in the Electronic Supplementary Material (ESM)).

As illustrated in Fig. 8(a), many discontinuous flaky films were observed in the SEM and HR-SEM images (marked by blue arrows) when the wear track surface was lubricated by the base liquid, demonstrating the poor lubrication ability of the base liquid. The direct contact of rubbing surfaces and the severe adhesive and corrosive wear should occur during the friction process. Fig. 8(b) shows that those flaky films disappeared for the wear track lubricated by BP dispersion. However, many deep scratches emerged, implying that severe abrasive wear occurred because of the poor dispersion stability of BP in water and its large particle size. A tight and smooth film on the wear track surface lubricated by the BPQDs dispersion (0.005 wt $\%$ ) was observed despite the presence of few small cracks. BPQDs are crucial in the formation of a boundary lubrication film, owing to their small and uniform particle size, good dispersion stability, and high reactivity. That film prevented the rubbing surfaces from direct contact, and hence reduced friction and wear.

Figure 9 illustrates the selected elemental area maps(from HR-SEM images) of wear track surfaces lubricated by the base liquid, the BP and BPQDs
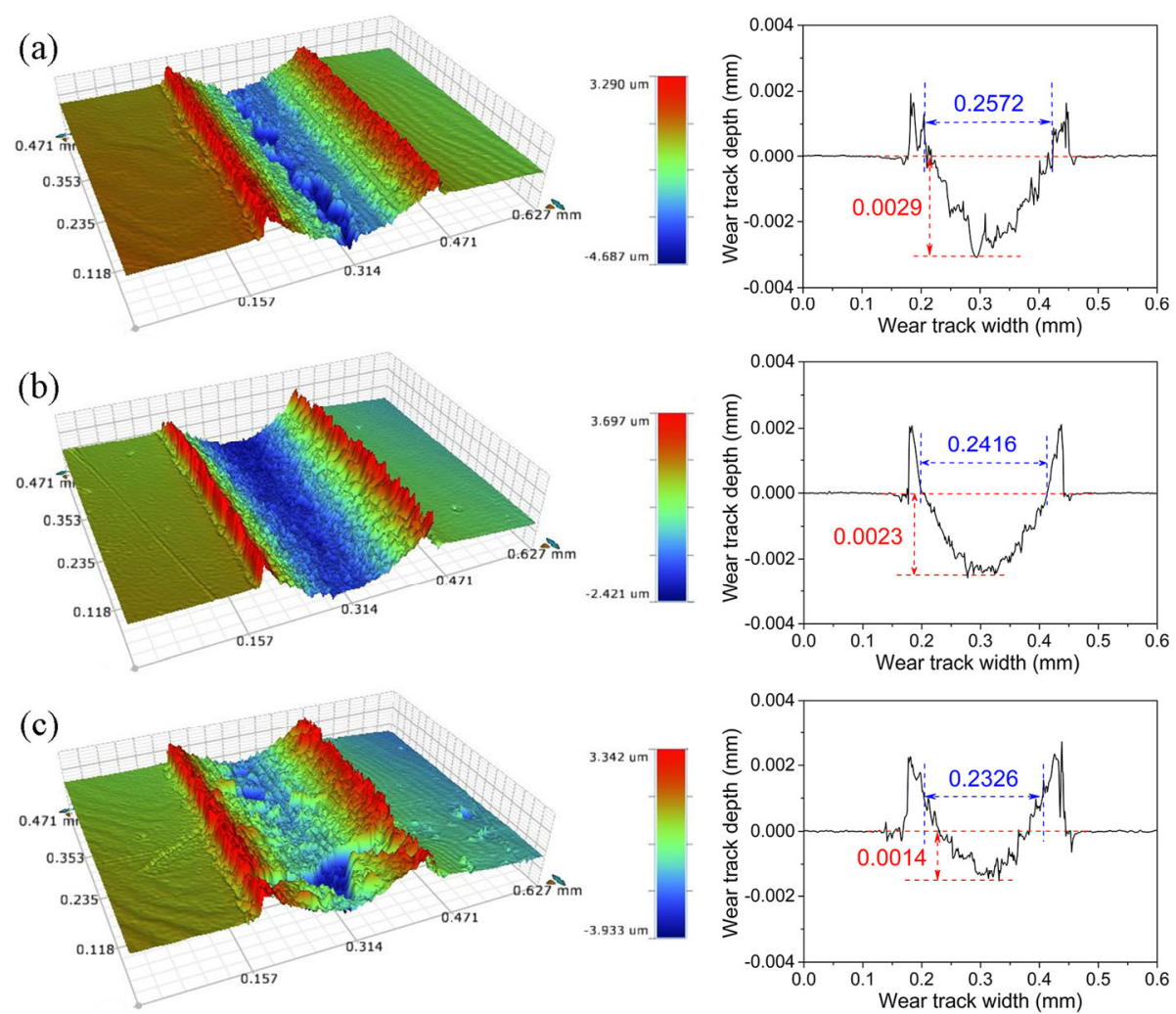

Fig. 7 3D morphologies and two-dimensional profiles of the wear tracks of lower plates lubricated by (a) base liquid, (b) $0.005 \mathrm{wt} \% \mathrm{BP}$ dispersion, and (c) $0.005 \mathrm{wt} \%$ BPQDs dispersion. (Test conditions: $5 \mathrm{~Hz}, 20 \mathrm{~min}, 40 \mathrm{~N}$, and room temperature). 


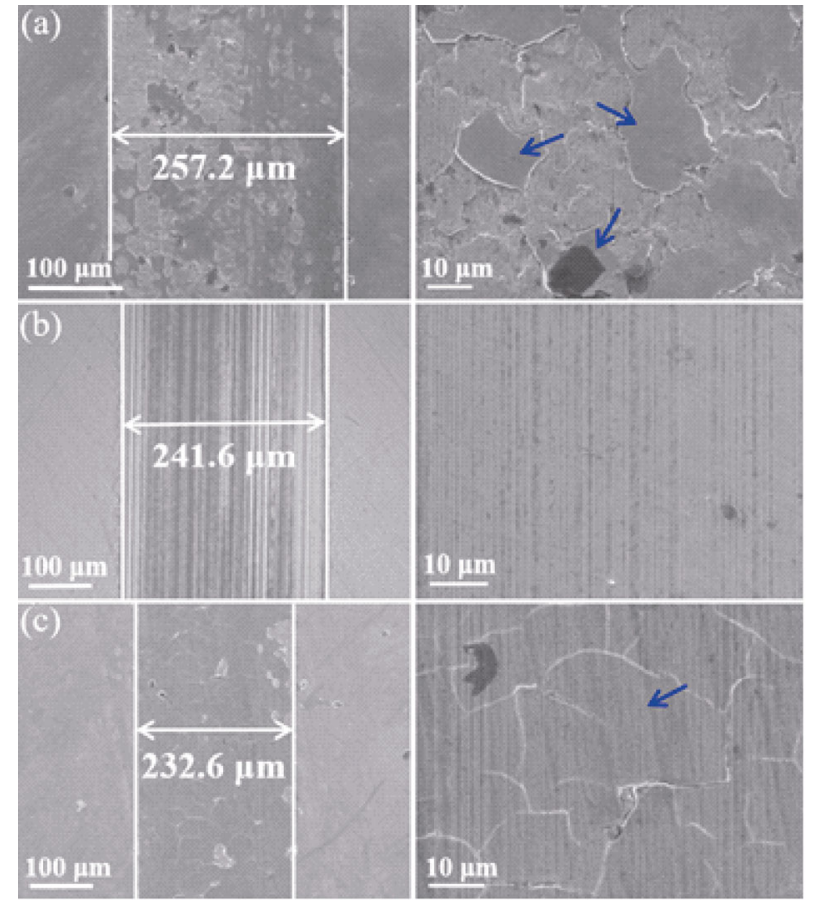

Fig. 8 Low-(left) and high-resolution (right) SEM images of the wear track surfaces lubricated by (a) base liquid, (b) 0.005 wt $\%$ BP dispersion, and (c) $0.005 \mathrm{wt} \%$ BPQDs dispersion. (Test conditions: $5 \mathrm{~Hz}, 20 \mathrm{~min}, 40 \mathrm{~N}$, and room temperature).

dispersions (0.005 wt\%). As shown in Fig. 9(a), the uniform distribution of $\mathrm{Fe}, \mathrm{C}, \mathrm{O}$, and $\mathrm{N}$ was confirmed on the wear track surface lubricated by the base liquid. The detected $\mathrm{C}$ and $\mathrm{N}$ were mainly stemmed from the adsorption of TEA molecules. In addition to those elements, a trace amount of $P$ was detected on the wear track surface lubricated by the $0.005 \mathrm{wt} \%$ BP dispersion (Fig. 9(b)), derived from remnant $\mathrm{BP}$ components during the friction process. As shown in Fig. 9(c), except for Fe, C, O and $\mathrm{N}$, a great amount of $\mathrm{P}$ was uniformly distributed on the wear track surface lubricated by the BPQDs dispersion, confirming that BPQDs can participate in the formation of the boundary tribofilm. The detailed element compositions and EDS spectra of wear track surfaces lubricated by the base liquid and dispersions are shown in Fig. S2 (in ESM). They were consistent with the results of the elemental maps. According to these findings, it can be inferred that the ultra-small BPQDs easily react with the rubbing surfaces under the high frictional heat and contact pressure to form a robust boundary tribofilm, which plays an important role on reducing the friction and wear impacts.

The full-scan XPS spectra results (Fig. S3 in ESM) confirmed that the elemental compositions of the wear track surfaces lubricated by the base liquid and the dispersions were consistent with the EDS analyses (Fig. S2 in ESM). Figure 10 shows the high-resolution (HR) elemental XPS spectra of the wear track surfaces at the lower plates lubricated by the base liquid, the BP dispersion (0.005 $\mathrm{wt} \%)$, and the BPQDs dispersion (0.005 wt \%). The HR Fe $2 \mathrm{p}, \mathrm{O} 1 \mathrm{~s}$, and C 1s XPS spectra in Fig. 10(a) demonstrated that iron oxides (such as $\mathrm{FeO}$ at 708.5/528.7 eV and $\mathrm{Fe}_{2} \mathrm{O}_{3}$ at 713.1/530.7 eV) and organic compounds (composed of $\mathrm{C}, \mathrm{O}$, and $\mathrm{N}$ ) can be probed on the wear track surface lubricated by the base liquid. The typical fitting elemental
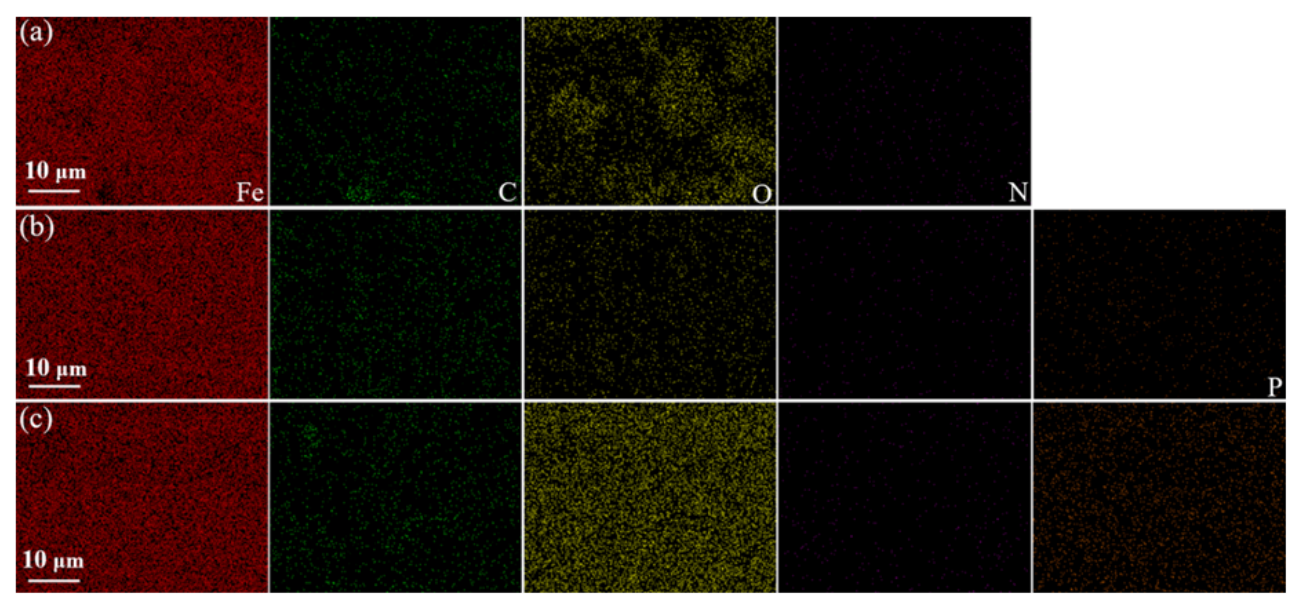

Fig. 9 Element maps of the wear track surfaces lubricated by: (a) base liquid, (b) $0.005 \mathrm{wt} \%$ BP dispersion, and (c) $0.005 \mathrm{wt} \%$ BPQDs dispersion. (Red: Fe, green: $\mathrm{C}$, yellow: O, purple: $\mathrm{N}$, and orange: $\mathrm{P}$ ). 
XPS peaks of the wear track surface lubricated by the BP dispersion (Fig. 10(b)) were similar to those of the base liquid, except for the fact that the $\mathrm{C}-\mathrm{N}$ species disappeared. This suggested that the abrasive wear of the BP particles hindered the absorption of TEA molecules on the rubbing surfaces. Moreover, no characteristic peaks were detected in the HR P $2 \mathrm{p}$ XPS spectrum (Fig. S4 in ESM). These results proved the poor embedding stability of BP between the rubbing surfaces, and hence low film-forming ability. In addition to the described peaks, some new peaks at $710.8 \mathrm{eV}$ (Fe 2p), $532.1 \mathrm{eV}$ (O 1s), $132.6 \mathrm{eV}$ (P 2p), $535.7 \mathrm{eV}$ (O 1s), $139.4 \mathrm{eV}$ (P 2p), and $283.1 \mathrm{eV}(\mathrm{C} 1 \mathrm{~s})$ that were attributed to the iron phosphate $\left(\mathrm{FePO}_{4}\right)$, phosphorus pentoxide $\left(\mathrm{P}_{2} \mathrm{O}_{5}\right)$, and metal carbide $(\mathrm{FeC})$ could be detected on the wear track surface lubricated by the BPQDs dispersion (Fig. 10(c)). These results clearly confirmed that the complicated tribochemical reactions between the rubbing surface and the BPQDs might take place during the friction process.
The cross-sectional TEM image in Fig. 11(a) demonstrates that a continuous, uniform, and tight tribofilm with a thickness of approximately $90 \mathrm{~nm}$ could be present between the Pt protection layer and the steel substrate on the wear track lubricated by the BPQDs dispersion (0.005 wt\%). As shown in Figs. 11(b) and 11(c), the formed tribofilm was almost amorphous and embedded with many nanocrystals with an average particle diameter of approximately $4.0 \mathrm{~nm}$. Additionally, the HR-TEM image (inset in Fig. 11(c)) demonstrates that the spacing of the lattice fringes of the nanocrystal was approximately $0.22 \mathrm{~nm}$, which is attributed to the (020) plane of the BP crystal. These results collectively indicate that the detected nanocrystals in the tribofilm are BPQDs. In Fig. 11(d), EDS elemental maps of the selected areas show that the tribofilm has high contents of $\mathrm{C}, \mathrm{O}, \mathrm{N}$, and $\mathrm{P}$, and a low content of $\mathrm{Fe}$, which is consistent with the SEM and XPS characterizations. It may be observed that the formed tribofilm was actually a tribochemical film
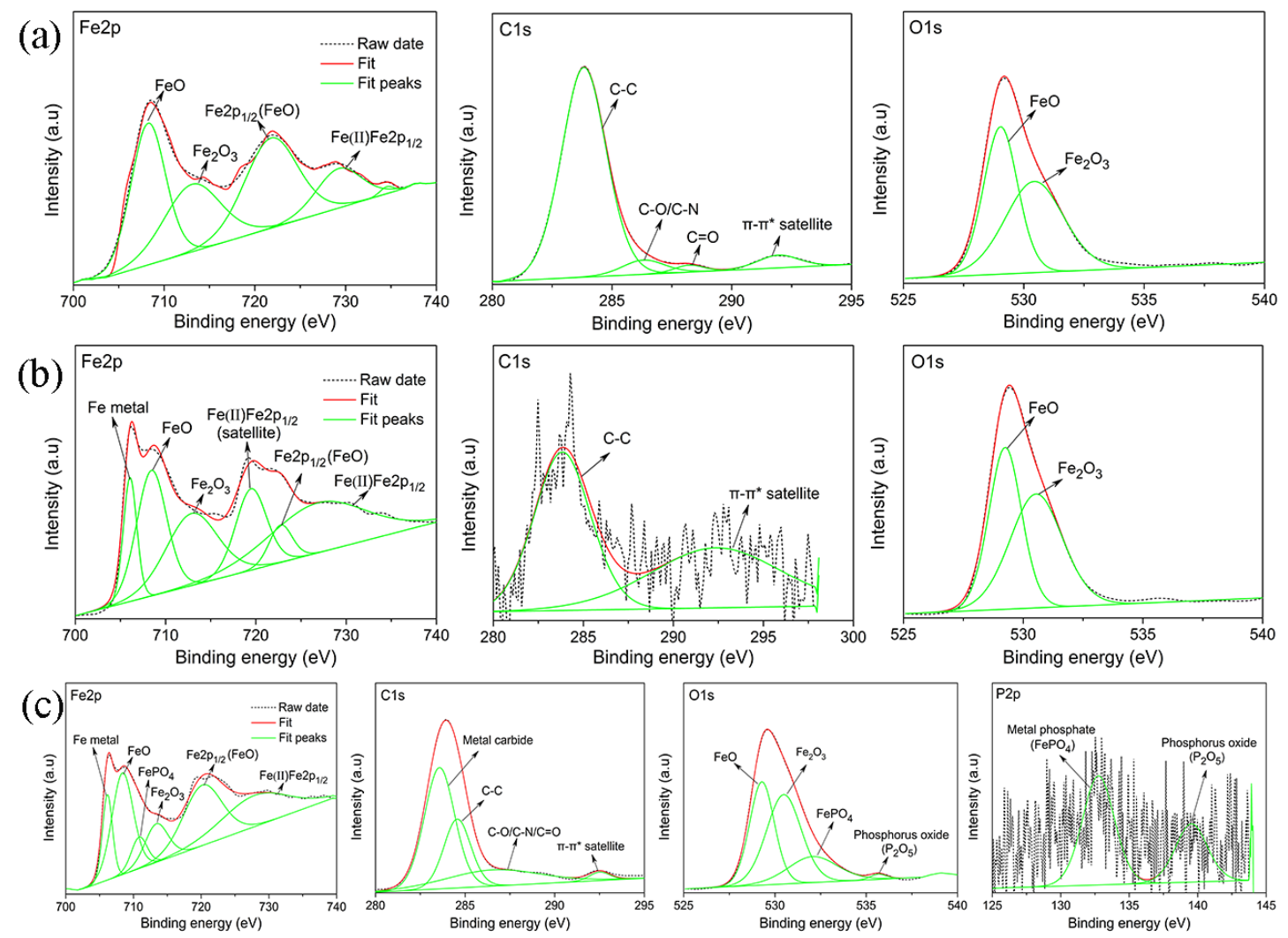

Fig. 10 (a) HR XPS Fe 2p, C 1s, and O 1s spectra of the wear track surface lubricated by the base liquid. (b) HR XPS Fe $2 p$, C $1 \mathrm{~s}$, and $\mathrm{O} 1 \mathrm{~s}$ spectra of the wear track surface lubricated by the $0.005 \mathrm{wt} \%$ BP dispersion. (c) HR XPS Fe $2 \mathrm{p}, \mathrm{C} 1 \mathrm{~s}, \mathrm{O} 1 \mathrm{~s}$, and P $2 \mathrm{p}$ spectra of the wear track surface lubricated by the $0.005 \mathrm{wt} \%$ BPQDs dispersion. 


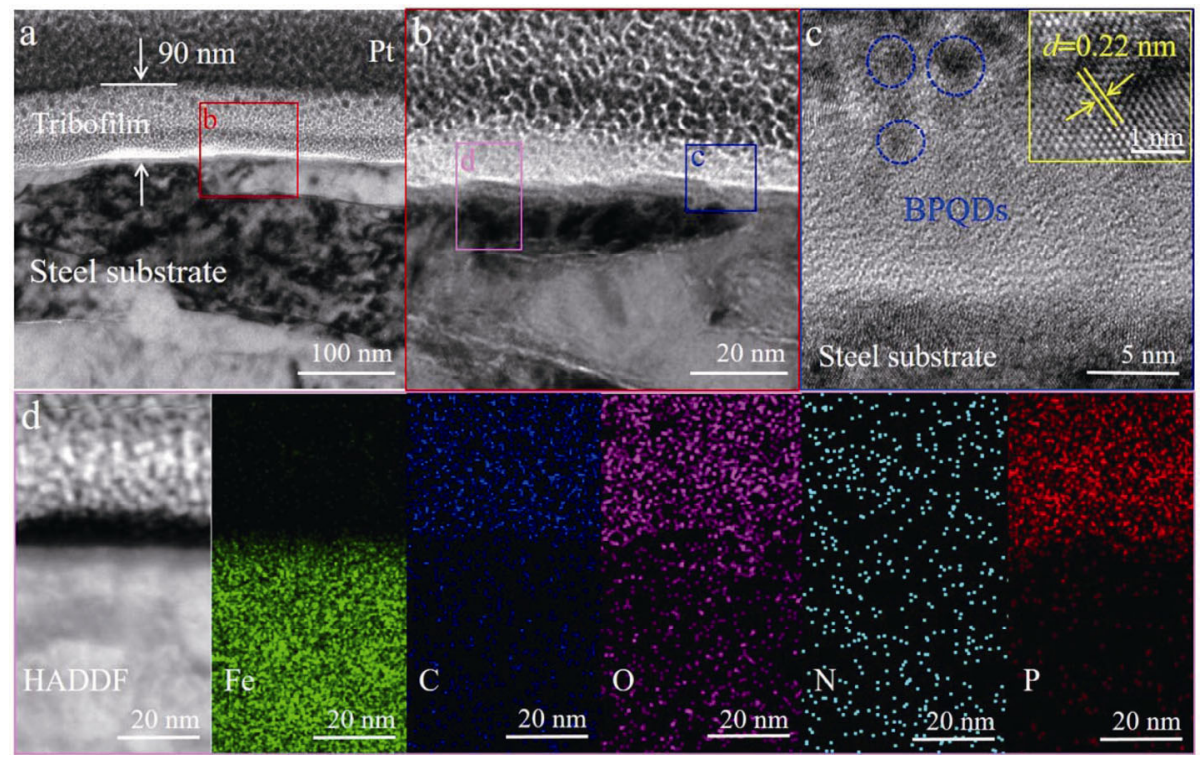

Fig. 11 (a-c) Bright-field cross-section TEM images and (d) EDS elemental maps of the wear scar of lower plate lubricated by the BPQDs dispersion (0.005 wt\%). (Test conditions: $5 \mathrm{~Hz}, 20 \mathrm{~min}, 40 \mathrm{~N}$, and room temperature).

embedded with BPQDs.

Based on the results of the wear track surface analysis, proposing a reasonable lubrication mechanism for $\mathrm{BP}$ and BPQDs as water-based lubricant additives (shown in Fig. 12) was attempted. As previously mentioned, the complicated tribochemical reactions between the rubbing surfaces and BPQDs should take place to form a sophisticated tribochemical film, which was observed in the corresponding brightfield cross-sectional TEM images (Fig. 11). The formed tribochemical film comprised iron oxides ( $\mathrm{FeO}$ and $\mathrm{Fe}_{2} \mathrm{O}_{3}$ ), iron phosphate $\left(\mathrm{FePO}_{4}\right)$, metal carbide $(\mathrm{FeC})$, phosphorus oxide $\left(\mathrm{P}_{2} \mathrm{O}_{5}\right)$, and organic compounds embedded with the BPQDs. The formed tribochemical film could effectively improve the friction-reducing and anti-wear effects of friction pairs by preventing the direct contact of rubbing surfaces [58, 63, 64]. Additionally, the BPQDs embedded into the tribochemical film may also function as ball-bearings, which provide nano-lubrication effects (such as mending, polishing and rolling effects) to further reduce friction and wear [14, 65]. BP neither exhibited the nano-lubrication effects due to its poor dispersion stability, nor reacted with the rubbing surface to form a tribochemical film because of its low reactivity. In addition, BP might be squeezed from rubbing surfaces during the friction process because of its large particle

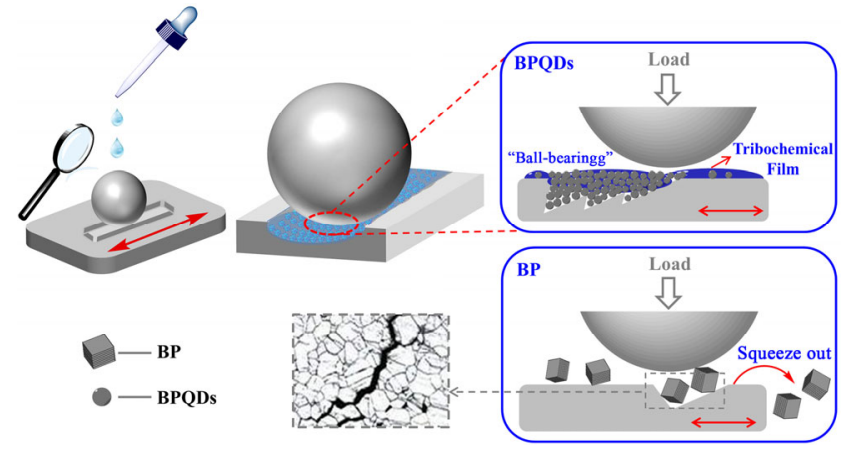

Fig. 12 Schematic of the lubrication mechanism of BPQDs and BP as water-based lubricant additives.

size, resulting in the direct contact with the roughness of the rubbing surfaces, hence having a limited tribological performance. In summary, the formed tribochemical film embedded with BPQDs during the friction process was in principle responsible for the superior tribological performance of BPQDs.

\section{Conclusions}

BPQDs with a rounded nanosheet morphology similar to graphene quantum dots (average particle size of $3.9 \mathrm{~nm}$ and thickness of $1.0 \mathrm{~nm}$ ) were successfully synthesized by the simple solution-based top-down method. The BPQDs had an excellent dispersion stability in water and were used as water-based lubricant additives. The BPQD-based additives 
exhibited remarkable friction-reducing and antiwear performances. At a very small additive content (c) of $0.005 \mathrm{wt} \%$, the BPQDs could reduce the friction coefficient and wear volume of the base liquid by $32.3 \%$ and $56.4 \%$, respectively. Meanwhile, the load-carrying capacity of the base liquid increased from $120 \mathrm{~N}$ to at least $300 \mathrm{~N}$. The tribological properties of BPQDs were slightly attenuated only when the friction test duration prolonged from 20 to $120 \mathrm{~min}$, and the load was increased from 40 to $80 \mathrm{~N}$. The tribological performance of BPQDs was distinctly superior to that of BP. The results of the wear-track surface analysis illustrated that BPQDs could react with the rubbing surface to form a robust tribochemical film with an average thickness of approximately $90 \mathrm{~nm}$, under local high frictional heat and high contact pressure, thus preventing direct contact between the rubbing surfaces and significantly reducing friction and wear. Additionally, BPQDs may also act as "ball-bearings" that provide mending, polishing, and rolling effects to further lower the friction and wear of rubbing surfaces. In summary, BPQDs as novel lubricant additives have shown high potential to be used in water-based lubrication.

Electronic Supplementary Material: Supplementary material is available in the online version of this article at https://doi.org/10.1007/s40544-020-04348.

Open Access This article is licensed under a Creative Commons Attribution 4.0 International License, which permits use, sharing, adaptation, distribution and reproduction in any medium or format, as long as you give appropriate credit to the original author(s) and the source, provide a link to the Creative Commons licence, and indicate if changes were made.

The images or other third party material in this article are included in the article's Creative Commons licence, unless indicated otherwise in a credit line to the material. If material is not included in the article's Creative Commons licence and your intended use is not permitted by statutory regulation or exceeds the permitted use, you will need to obtain permission directly from the copyright holder.

To view a copy of this licence, visit http://creativecommons.org/licenses/by/4.0/.

\section{References}

[1] Xiao H, Liu S, Xu Q, Zhang H. Carbon quantum dots: An innovative additive for water lubrication. Sci China Technol Sci 62(4): 587-596 (2018)

[2] Kogovšek J, Kalin M. Various $\mathrm{MoS}_{2^{-}}, \mathrm{WS}_{2}$-and C-based micro-and nanoparticles in boundary lubrication. Tribol Lett 53(3): 585-597 (2014)

[3] Spear J C, Ewers B W, Batteas J D. 2D-nanomaterials for controlling friction and wear at interfaces. Nano Today 10(3): 301-314 (2015)

[4] Zhai W, Srikanth N, Kong L B, Zhou K. Carbon nanomaterials in tribology. Carbon 119: 150-171 (2017)

[5] Jaiswal V, Kalyani, Umrao S, Rastogi R B, Kumar R, Srivastava A. Synthesis, characterization, and tribological evaluation of $\mathrm{TiO}_{2}$-reinforced boron and nitrogen co-doped reduced graphene oxide based hybrid nanomaterials as efficient antiwear lubricant additives. ACS Appl Mater Inter 8(18): 11698-11710 (2016)

[6] Berman D, Erdemir A, Sumant A V. Graphene: a new emerging lubricant. Mater Today 17(1): 31-42 (2014)

[7] He X, Xiao H, Choi H, Díaz A, Mosby B, Clearfield A, Liang H. $\alpha$-Zirconium phosphate nanoplatelets as lubricant additives. Colloid Surf A: Phys Eng Aspect 452: 32-38 (2014)

[8] Wang K, Wu H, Wang H, Liu Y, Yang L, Zhao L. Tribological properties of novel palygorskite nanoplatelets used as oil-based lubricant additives. Friction 9: 332-343 (2021)

[9] An L, Yu Y, Bai C, Bai Y, Zhang B, Gao K, Wang X, Lai Z, Zhang J. Simultaneous production and functionalization of hexagonal boron nitride nanosheets by solvent-free mechanical exfoliation for superlubricant water-based lubricant additives. $2 D$ Mater Appl 3(1): 1-9 (2019)

[10] Mai Y, Li Y, Li S, Zhang L, Liu C, Jie X. Selflubricating Ti3C2 nanosheets/copper composite coatings. J Alloy Compound 770: 1-5 (2019)

[11] Kumari S, Chouhan A, Khatri O P. Nanostructured layered materials as novel lubricant additives for tribological applications. Tribology in Materials and Applications Springer, 2020: 157-178.

[12] Xiao H, Liu S. 2D nanomaterials as lubricant additive: A review. Mater Design 135: 319-332 (2017)

[13] Liu L, Zhou M, Li X, Jin L, Su G, Mo Y, Li L, Zhu H, Tian Y. Research progress in application of 2D materials in liquid-phase lubrication system. Materials 11(8): 1314 (2018) 
[14] Dai W, Kheireddin B, Gao H, Liang H. Roles of nanoparticles in oil lubrication. Tribol Int 102: 88-98 (2016)

[15] Gulzar M, Masjuki H H, Kalam M A, Varman M, Zulkifli N W M, Mufti R A, Zahid R. Tribological performance of nanoparticles as lubricating oil additives. J Nanopart Res 18(8): 223-248 (2016)

[16] Wang W, Xie G, Luo J. Black phosphorus as a new lubricant. Friction 6(1): 116-142 (2018)

[17] Cui Z, Xie G, He F, Wang W, Guo D, Wang W. Atomic-scale friction of black phosphorus: effect of thickness and anisotropic behavior. Adv Mater Interfaces 4(23): 1700998 (2017)

[18] Tang G, Su F, Xu X, Chu P K. 2D black phosphorus dotted with silver nanoparticles: An excellent lubricant additive for tribological applications. Chem Eng $J$ 392: 123631 (2019)

[19] Guo P, Qi S, Chen L, Gou C, Lin B, Lu Z, Wu Z, Zhang G. Black phosphorus-graphene oxide hybrid nanomaterials toward advanced lubricating properties under water. Adv Mater Interfaces 6(23): 1901174 (2019)

[20] Wu S, He F, Xie G, Bian Z, Luo J, Wen S. Black phosphorus: degradation favors lubrication. Nano Lett 18(9): 5618-5627 (2018)

[21] Wang W, Xie G, Luo J. Superlubricity of black phosphorus as lubricant additive. ACS Appl Mater Inter 10(49): 43203-43210 (2018)

[22] Peng S, Guo Y, Xie G, Luo J. Tribological behavior of polytetrafluoroethylene coating reinforced with black phosphorus nanoparticles. Appl Surf Sci 441: 670-677 (2018)

[23] Lv Y, Wang W, Xie G, Luo J. Self-lubricating PTFEbased composites with black phosphorus nanosheets. Tribol Lett 66(2): 61-72 (2018)

[24] Zhang X, Xie H, Liu Z, Tan C, Luo Z, Li H, Lin J, Sun L, Chen W, Xu Z, Xie L, Huang W, Zhang H. Black phosphorus quantum dots. Angew Chem Int Ed 54(12): 3653-3657 (2015)

[25] Sun Z, Zhao Y, Li Z, Cui H, Zhou Y, Li W, Tao W, Zhang H, Wang H, Chu P K, Yu X F. TiL 4 -coordinated black phosphorus quantum dots as an efficient contrast agent for in vivo photoacoustic imaging of cancer. Small 13(11): 1602896 (2017)

[26] Sun C, Wen L, Zeng J, Wang Y, Sun Q, Deng L, Zhao C, $\mathrm{Li}$ Z. One-pot solventless preparation of PEGylated black phosphorus nanoparticles for photoacoustic imaging and photothermal therapy of cancer. Biomaterials 91: 81-89 (2016)

[27] Zhang Z, Li Y, Xu J, Wen Y. Electropolymerized molecularly imprinted polypyrrole decorated with black phosphorene quantum dots onto poly $(3,4-$ ethylenedioxythiophene) nanorods and its voltammetric sensing of vitamin C. J Electroanal Chem 814: 153-160 (2018)

[28] Gu W, Yan Y, Pei X, Zhang C, Ding C, Xian Y. Fluorescent black phosphorus quantum dots as label-free sensing probes for evaluation of acetylcholinesterase activity. Sensor Actuator B: Chem 250: 601-607 (2017)

[29] Gu W, Pei X, Cheng Y, Zhang C, Zhang J, Yan Y, Ding C, Xian Y. Black phosphorus quantum dots as the ratiometric fluorescence probe for trace mercury ion detection based on inner filter effect. ACS sensors 2(4): 576-582 (2017)

[30] Yin F, Hu K, Chen S, Wang D, Zhang J, Xie M, Yang D, Qiu M, Zhang H, Li Z-g. Black phosphorus quantum dot based novel siRNA delivery systems in human pluripotent teratoma PA-1 cells. J Mater Chem B 5(27): 5433-5440 (2017)

[31] Li Y, Liu Z, Hou Y, Yang G, Fei X, Zhao H, Guo Y, Su C, Wang Z, Zhong H, Zhuang Z, Guo Z. Multifunctional nanoplatform based on black phosphorus quantum dots for bioimaging and photodynamic/photothermal synergistic cancer therapy. ACS Appl Mater Inter 9(30): 25098-25106 (2017)

[32] Shao J, Xie H, Huang H, Li Z, Sun Z, Xu Y, Xiao Q, Yu $\mathrm{X}$ F, Zhao $\mathrm{Y}$, Zhang $\mathrm{H}$, Wang $\mathrm{H}$, Chu $\mathrm{P} \mathrm{K}$. Biodegradable black phosphorus-based nanospheres for in vivo photothermal cancer therapy. Nat Commun 7: 12967-12980 (2016)

[33] Fu N, Huang C, Lin P, Zhu M, Li T, Ye M, Lin S, Zhang G, Du J, Liu C, Xu B, Wang D, Ke S. Black phosphorus quantum dots as dual-functional electron-selective materials for efficient plastic perovskite solar cells. $J$ Mater Chem A 6(19): 8886-8894 (2018)

[34] Li X, Li F, Lu X, Zuo S, Zhuang Z, Yao C. Black phosphorus quantum dots/attapulgite nanocomposite with enhanced photocatalytic performance. Funct Mater Lett 10(06): 1750078 (2018)

[35] Lei W, Mi Y, Feng R, Liu P, Hu S, Yu J, Liu X, Rodriguez J A, Wang J O, Zheng L, Tang K, Zhu S, Liu G, Liu M. Hybrid 0D-2D black phosphorus quantum dots-graphitic carbon nitride nanosheets for efficient hydrogen evolution. Nano Energy 50: 552-561 (2018)

[36] Gui R, Jin H, Wang Z, Li J. Black phosphorus quantum dots: synthesis, properties, functionalized modification and applications. Chem Soc Rev 47(17): 6795-6823 (2018)

[37] Tang J, Chen S, Jia Y, Ma Y, Xie H, Quan X, Ding Q. Carbon dots as an additive for improving performance in water-based lubricants for amorphous carbon (a-C) coatings. Carbon 156: 272-281 (2020)

[38] Qiang R, Hu L, Hou K, Wang J, Yang S. Water-soluble 
graphene quantum dots as high-performance waterbased lubricant additive for steel/steel contact. Tribol Lett 67(2): 64 (2019)

[39] Liu X, Chen Y. Synthesis of polyethylene glycol modified carbon dots as a kind of excellent water-based lubricant additives. Fuller Nanotub Car $N$ 27(5): 400-409 (2019)

[40] He C, Yan H, Wang X, Bai M. Graphene quantum dots prepared by gaseous detonation toward excellent friction-reducing and antiwear additives. Diam Relat Mater 89: 293-300 (2018)

[41] Wu X H, Gong K L, Zhao G Q, Lou W J, Wang X B, Liu W M. $\mathrm{MoS}_{2} / \mathrm{WS}_{2}$ quantum dots as high-performance lubricant additive in polyalkylene glycol for steel/steel contact at elevated temperature. Adv Mater Interfaces 5(1): 1700859 (2018)

[42] Ren X, Yang X, Xie G, Luo J. Black phosphorus quantum dots in aqueous ethylene glycol for macroscale superlubricity. ACS Appl Nano Mater 3(5): 4799-4809 (2020)

[43] Zhao W, Xue Z, Wang J, Jiang J, Zhao X, Mu T. Large-scale, highly efficient, and green liquidexfoliation of black phosphorus in ionic liquids. ACS Appl Mater Inter 7(50): 27608-27612 (2015)

[44] Lu H, Tang W, Liu X, Wang B, Huang Z. Oleylamine-modified carbon nanoparticles as a kind of efficient lubricating additive of polyalphaolefin. $J$ Mater Sci 52(8): 4483-4492 (2016)

[45] Mou Z, Wang B, Lu H, Quan H, Huang Z. Branched polyelectrolyte grafted carbon dots as the highperformance friction-reducing and antiwear additives of polyethylene glycol. Carbon 149: 594-603 (2019)

[46] Kumara C, Luo H, Leonard D N, Meyer H M, Qu J. Organic-modified silver nanoparticles as lubricant additives. ACS Appl Mater Inter 9: 37227-37237 (2017)

[47] Luo T, Wei X, Huang X, Huang L, Yang F. Tribological properties of $\mathrm{Al}_{2} \mathrm{O}_{3}$ nanoparticles as lubricating oil additives. Ceram Int 40(5): 7143-7149 (2014)

[48] Wu H, Zhao J, Cheng X, Xia W, He A, Yun J H, Huang S, Wang L, Huang H, Jiao S, Jiang Z. Friction and wear characteristics of $\mathrm{TiO}_{2}$ nano-additive water-based lubricant on ferritic stainless steel. Tribol Int 117: 24-38 (2018)

[49] Nan F, Zhou K, Liu S, Pu J, Fang Y, Ding W. Tribological properties of attapulgite $/ \mathrm{La}_{2} \mathrm{O}_{3}$ nanocomposite as lubricant additive for a steel/steel contact. $R S C A d v$ 8(30): 16947-16956 (2018)

[50] Wu X H, Gong K L, Zhao G Q, Lou W J, Wang X B, Liu W M. $\mathrm{MoS}_{2} / \mathrm{WS}_{2}$ quantum dots as high-performance lubricant additive in polyalkylene glycol for steel/steel contact at elevated temperature. Adv Mater Interfaces 5(1): 1700859 (2018)
[51] Rajendhran N, Palanisamy S, Periyasamy P, Venkatachalam R. Enhancing of the tribological characteristics of the lubricant oils using Ni-promoted $\mathrm{MoS}_{2}$ nanosheets as nano-additives. Tribol Int 118: 314-28 (2018)

[52] Wu P R, Kong Y C, Ma Z S, Ge T, Feng Y M, Liu Z, Cheng $Z$ L. Preparation and tribological properties of novel zinc borate/ $\mathrm{MoS}_{2}$ nanocomposites in grease. $J$ Alloy Compound 740: 823-829 (2018)

[53] Li Z, Ma S, Zhang G, Wang D, Zhou F. Soft/hard-coupled amphiphilic polymer nanospheres for water lubrication. ACS Appl Mater Inter 10(10): 9178-9187 (2018)

[54] Alazemi A A, Etacheri V, Dysart A D, Stacke L E, Pol V G, Sadeghi F. Ultrasmooth submicrometer carbon spheres as lubricant additives for friction and wear reduction. ACS Appl Mater Inter 7(9): 5514-5521 (2015)

[55] Zhang G Q, Zeng X Q, Ren T H, van der Heide E. Tribological properties of graphene oxide sheets as water-based lubricant additive. Ind Lubr Tribol 70(6): 1025-1036 (2018)

[56] Su F, Chen G, Huang P. Lubricating performances of graphene oxide and onion-like carbon as water-based lubricant additives for smooth and sand-blasted steel discs. Friction 8(1): 47-57 (2020)

[57] Shang W, Ye M, Cai T, Zhao L, Zhang Y, Liu D, Liu S. Tuning of the hydrophilicity and hydrophobicity of nitrogen doped carbon dots: A facile approach towards high efficient lubricant nanoadditives. J Mol Liq 266: 65-74 (2018)

[58] Hu Y, Wang Y, Wang C, Ye Y, Zhao H, Li J, Lu X, Mao C, Chen S, Mao J, Wang L, Xue Q. One-pot pyrolysis preparation of carbon dots as eco-friendly nanoadditives of water-based lubricants. Carbon 152: 511-520 (2019)

[59] Zhao L, Cai T, Ye M, Liu D, Liu S. The regulation of the microstructure, luminescence and lubricity of multi-element doped carbon nanodots with alkylated diquaternary 1,4-diazabicyclo[2.2.2]octane derived dicationic ionic liquids inserted in carbon skeleton. Carbon 150: 319-333 (2019)

[60] Li X F, Yan H, Peng S X. Colloidal polystyrene-carbon nanotubes as water-based lubricant additive. Adv Mater Res 228-229: 253-258 (2011)

[61] Ye X, Ma L, Yang Z, Wang J, Wang H, Yang S. Covalent functionalization of fluorinated graphene and subsequent application as water-based lubricant additive. ACS Appl Mater Inter 8(11): 7483-7488 (2016)

[62] Xie H, Jiang B, Dai J, Peng C, Li C, Li Q, Pan F. Tribological behaviors of graphene and graphene oxide 
as water-based lubricant additives for magnesium alloy/steel contacts. Materials 11(2): 206 (2018)

[63] Zhou Y, Qu J. Ionic liquids as lubricant additives: A review. ACS Appl Mater Inter 9(4): 3209-3222 (2017)

[64] Tu Z, Hu E, Wang B, David K D, Seeger P, Moneke M, Stengler $\mathrm{R}, \mathrm{Hu} \mathrm{K}, \mathrm{Hu} \mathrm{X}$. Tribological behaviors of

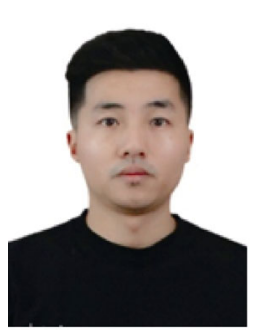

Weiwei TANG. He received his Ph.D. degrees in chemical engineering and technology from Southwest Petroleum University, Chengdu, China, in 2019. He joined

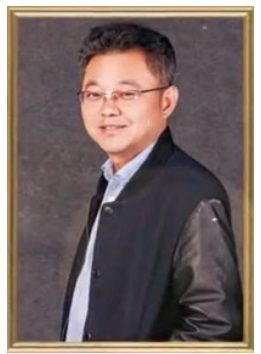

Yufeng LI. He received his M.S. degree in applied chemistry from Southwest Petroleum University, Chengdu, China, in 2006, and Ph.D. degree in material physics and chemistry from Sichuan University, Chengdu, China, in
Ni-modified citric acid carbon quantum dot particles as a green additive in polyethylene glycol. Friction 8(1): 182-197 (2020)

[65] He C, Yan H, Li X, Wang X. In situ fabrication of carbon dots-based lubricants using a facile ultrasonic approach. Green Chem 21(9): 2279-2285 (2019)

the School of Biological and Chemical Engineering at Panzhihua University from 2019. His current position is a lecturer. His research interests include nanomaterials, nanocomposites, and microtribology.

2013, respectively. He joined the School of Biological and Chemical Engineering at Panzhihua University from 1997. His current position is a professor. His research areas cover the carbon materials, nanocomposites, and lithium-ion batteries. 